Pacific Journal of Mathematics

FOR THE REDUCED WAVE ABSORPTION PRINCIPI TOSHES SAND 


\section{A REMARK ON THE \\ LIMITING ABSORPTION PRINCIPLE \\ FOR THE REDUCED WAVE EQUATION \\ WITH TWO UNBOUNDED MEDIA}

\section{Yoshimi SaItõ}

Eidus recently proved the limiting absorption principle for the reduced wave equation with two unbounded media, and he used it to show the limiting amplitude principle for the wave equation. In this paper we shall show that his limiting absorption principle can be improved so that it holds on the same weighted Sobolev spaces as were used in the case of the Schrödinger equation.

1. Introduction. Let us consider the reduced wave operator

$$
H u=-\mu(x)^{-1} \Delta
$$

in $\mathbf{R}^{N}$, where $\Delta$ is the Laplacian in $\mathbf{R}^{N}$ and $\mu(x)$ is a positive function in $\mathbf{R}^{N}$. The operator $H$ can be regarded as a selfadjoint operator in the Hilbert space $\mathscr{H}$ of all measurable functions $f(x)$ on $\mathbf{R}^{N}$ such that $f(x) \sqrt{\mu(x)}$ is square integrable over $\mathbf{R}^{N}$ (see $\S 2$ ). The reduced wave operator $H$ is obtained from the wave equation

$$
\mu(x) \frac{\partial^{2} w}{\partial t^{2}}-\Delta w=0
$$

by separation of the time variable $t$.

Through this work it is assumed that the function $\mu(x)$ on $\mathbf{R}^{N}$ with $N \geq 2$ has the form

$$
\mu(x)=\mu_{l} \quad\left(x \in \Omega_{l}, l=1,2\right),
$$

with positive constants $\mu_{l}\left(\mu_{1} \neq \mu_{2}\right)$ and disjoint open sets $\Omega_{l}, l=1,2$, given by

$$
\begin{aligned}
& \Omega_{1}=\left\{x \in \mathbf{R}^{N} / x_{N}>\varphi\left(x^{\prime}\right)\right\} \quad \text { and } \\
& \Omega_{2}=\left\{x \in \mathbf{R}^{N} / x_{N}<\varphi\left(x^{\prime}\right)\right\},
\end{aligned}
$$

where $x=\left(x^{\prime}, x_{N}\right), x^{\prime}=\left(x_{1}, x_{2}, \ldots, x_{N-1}\right)$ and $\varphi \in C^{1}\left(\mathbf{R}^{N-1} \backslash\{0\}\right)$. The separating surface $S$ is defined by

$$
S=\left\{x \in \mathbf{R}^{N} / x_{N}=\varphi\left(x^{\prime}\right)\right\}
$$


and $n_{l}(x)=\left(n_{l 1}(x), n_{l 2}(x), \ldots, n_{l N}(x)\right), l=1,2$, denote the outward unit normal of the boundary $\partial \Omega_{l}$ of $\Omega_{l}$ at $x \in S \backslash\{0\}$.

Eidus [4] proved that the limiting absorption principle and the limiting amplitude principle hold for the reduced wave operator $H$ and the wave equation (1.2), respectively, under the following conditions on the surface $S$ :

Assumption 1.1. The separating surface $S$ has a "cone-like" shape in the following sense: Let $n_{l}(x)$ be as above. Then,

(i) the $N$ th component $n_{l N}(x)$ of $n_{l}(x)$ satisfies

$$
\left|n_{l N}(x)\right|>c_{1} \quad(l=1,2, x \in S \backslash\{0\})
$$

with a positive constant $c_{1}$

(ii) and we have

$$
\left|x \cdot n_{l}(x)\right| \leq c_{2} \quad(l=1,2, x \in S \backslash\{0\})
$$

with a positive constant $c_{2}$, where $x \cdot n_{l}(x)$ means the inner product of the vectors $x$ and $n_{l}(x)$ in $\mathbf{R}^{N}$.

Let us define the resolvent $R(z)$ of the operator $H$ by

$$
R(z)=(H-z)^{-1}
$$

for $z \in \mathbf{C} \backslash \mathbf{R}$. The resolvent $R(z)$ is a bounded linear operator on the Hilbert space $\mathscr{H}$ which is now equivalent to the usual $L_{2}$ space $L_{2}\left(\mathbf{R}^{N}\right)$. Let us introduce the weighted $L_{2}$ space $L_{2, \beta}\left(\mathbf{R}^{N}\right)$ by

$$
L_{2, \beta}\left(\mathbf{R}^{N}\right)=\left\{f(x) / \int_{\mathbf{R}^{N}}(1|x|)^{2 \beta}|f(x)|^{2} d x<\infty\right\}
$$

with its norm

$$
\|f\|_{\beta}=\left[\int_{\mathbf{R}^{N}}(1+|x|)^{2 \beta}|f(x)|^{2} d x\right]^{1 / 2} .
$$

where $\beta$ is a real number. (In Eidus [4], $L_{2, \beta}\left(\mathbf{R}^{N}\right)$ and \|\|$_{\beta}$ are denoted as $L_{2 \beta}^{2}$ and \|\|$_{2 \beta}$, respectively.) Then Eidus' result on the limiting absorption principle is stated as follows:

THEOREM 1.2 (Eidus [4], Theorem 3.2). Let Assumption 1.1 be satisfied. Then there exist the limits

$$
\lim _{\eta \rightarrow \pm 0} R(\lambda+i \eta)=R^{ \pm}(\lambda) \quad \text { in } B\left(L_{2,1}\left(\mathbf{R}^{N}\right), L_{2,-1}\left(\mathbf{R}^{N}\right)\right)
$$


for each $\lambda>0$, where $B(X, Y)$ is the Banach space of all bounded, linear operators from $X$ into $Y$. Furthermore, the limit $R^{ \pm}(\lambda)$ are Hölder continuous in $\lambda$ in the topology of $B\left(L_{2,1}\left(\mathbf{R}^{N}\right), L_{2,-1}\left(\mathbf{R}^{N}\right)\right)$.

The limiting absorption principle for partial differential operators has been studied for about twenty years. Especially many works have been done for Schrödinger operators

$$
T=-\Delta+V(x)
$$

in $\mathbf{R}^{N}$ to find various sufficient conditions on the potential $V(x)$ that the limiting absorption principle holds for $T$. Among them, we refer to Jäger [6], Saitō [10], Agmon [1] for the short-range potential, Ikebe \& Saitō [5], Lavine [8] for the long-range potential. Mochizuki \& Uchiyama [9], Devinatz \& Rejto [2] for the oscillatory long-range potential. It is shown by these works that under certain conditions on $V(x)$ there exist the limits

$$
\lim _{\eta \rightarrow \pm 0} R_{T}(\lambda+i \eta)=R_{T}^{ \pm}(\lambda) \quad \text { in } B\left(L_{2, \delta}\left(\mathbf{R}^{N}\right), L_{2,-\delta}\left(\mathbf{R}^{N}\right)\right),
$$

where $R_{T}(z)=(T-z)^{-1}, \lambda$ belongs to the continuous spectrum of $T$, and $\delta$ is a constant such that

$$
\delta>1 / 2
$$

The condition (1.14) is in a sense best possible, because, in general, $u=R_{T}^{ \pm}(\lambda) f$ does not belong to $L_{2,-\beta}\left(\mathbf{R}^{N}\right)$ for $\beta \leq 1 / 2$.

In this work we shall prove some new estimates (Propositions 3.3, $3.8,3.10$ etc.) for $u=R(z) f$ which, combined with the methods and results of Eidus [4], enable us to show, under Assumption 1.1, that for a set $K$ in $\mathbf{C} \backslash \mathbf{R}$ of the form

$$
K=\left\{z=\lambda+i \eta \in \mathbf{C} / \lambda_{0} \leq \lambda \leq \lambda_{1}, 0<|\eta| \leq \eta_{0}\right\}
$$

with positive constants $\lambda_{0}<\lambda_{1}$ and $\eta_{0}$ there exists a positive constant $C=C(K)$ depending only on $K$ such that

$$
\|R(z) f\|_{2,-\delta} \leq C\|f\|_{\delta} \quad\left(z \in K, f \in L_{2, \delta}\left(\mathbf{R}^{N}\right)\right),
$$

where $\delta$ is a constant satisfying (1.14) and \|\|$_{2,-\delta}$ denotes the norm of the wieghted Sobolev space $H_{-\delta}^{2}\left(\mathbf{R}^{N}\right)$ of all functions $u$ such that all the derivatives up to the second order belong to the weighted $L_{2}$ space $L_{2,-\delta}\left(\mathbf{R}^{N}\right)$ (cf. Eidus [4], Corollary 2.2). It is easy to see from (1.16) and Theorem 1.1 that the limits

$$
\text { s- } \lim _{\eta \rightarrow \pm 0} R(\lambda+i \eta) f=R^{ \pm}(\lambda) f \quad \text { in } H_{-\delta}^{2}\left(\mathbf{R}^{N}\right)
$$

exist for all $f \in L_{2, \delta}\left(\mathbf{R}^{N}\right)$, where s-lim means the strong limit. 
Let us explain our main idea. When we studied the limiting absorption principle for various Schrödinger operators, the classical Sommerfeld radiation condition

$$
\frac{\partial u}{\partial r}-i k u=\text { small at infinity }
$$

or its modifications played a very important role (e.g., Jäger [6], Saitō [10], Ikebe \& Saitō [5], Mochizuki \& Uchiyama [9], Saitō [11], [12]). First, as is well-known, the radiation condition guarantees the uniqueness of the solution. At the same time it has been known that we can show the limiting absorption principle through a priori estimates of the radiation condition. It will be seen in our case that it is useful for getting the estimate (1.16) to introduce a "modified radiation condition-like" term which contains a surface integral over the separating surface $S$ given by (1.5). The limiting absorption principle for the operator $H$ will be obtained through estimating the above "modified radiation condition-like" term. However, it seems that we need further investigation to see whether our radiation condition fully guarantees the uniqueness of the solution.

In $\S 2$ some basic a priori estimates on $u=R(z) f$, which were obtained by Eidus [4], will be given. In $\S 3$ we shall prove some more a priori estimates on the "modified radiation condition-like" term and $u=R(z) f$. The estimate (1.16) and the limiting absorption principle will be shown in $\S 4$.

2. A priori estimates for $u=R(z) f$. In this section we shall give some a priori estimates for $u=R(z) f$ with $z \in \mathbf{C} \backslash \mathbf{R}$ and $f \in L_{2}\left(\mathbf{R}^{N}\right)$, $N \geq 2$. All these estimates except for Lemma 2.4 are proved in Eidus [4].

Let us now call some usual notation for some function spaces which will be used in the sequel. Let $m$ and $\beta$ be a nonnegative integer and a real number, respectively, and let $G$ be an open set in $\mathbf{R}^{N}$. Then the weighted Sobolev space $H_{\beta}^{m}(G)$ is defined by

$$
H_{\beta}^{m}(G)=\left\{v \in \mathscr{D}^{\prime}(G) /(1+|x|)^{\beta} \partial^{\alpha} v \in L_{2}(G),|\alpha| \leq m\right\},
$$

where $\mathscr{D}^{\prime}(G)$ is all distributions on $G, \alpha=\left(\alpha_{1}, \alpha_{2}, \ldots, \alpha_{N}\right)$ is a multiindex with $|\alpha|=\alpha_{1}+\alpha_{2}+\cdots+\alpha_{N}$ and

$$
\partial^{\alpha} v=\frac{\partial^{|\alpha|} v}{\partial_{1}^{\alpha_{1}} \partial_{2}^{\alpha_{2}} \cdots \partial_{N}^{\alpha_{N}}}
$$

with

$$
\partial_{j}^{\alpha_{\jmath}}=\left(\frac{\partial}{\partial x_{j}}\right)^{\alpha_{J}} \quad(j=1,2, \ldots, N)
$$


The space $H_{\beta}^{m}(G)$ is a Hilbert space with its inner product

$$
(v, w)_{m, \beta, G}=\sum_{|\alpha| \leq m} \int_{G}(1+|x|)^{2 \beta}\left(\partial^{\alpha} v\right) \overline{\left(\partial^{\alpha} w\right)} d x
$$

and norm

$$
\|v\|_{m, \beta, G}=\left[(v, v)_{m, \beta, G}\right]^{1 / 2}
$$

We set

$$
H_{\beta}^{0}(G)=L_{2, \beta}(G),
$$

and the subscript 0 in $(,)_{0, \beta, G}$ or \|\|$_{0, \beta, G}$ will be omitted as in $(,)_{\beta, G}$ or \|\|$_{\beta, G}$. When $G=\mathbf{R}^{N}$, the subscript $G$ will be also omitted as in $(,)_{\beta}$. Let $\mathscr{H}$ be the Hilbert space defined by

$$
\mathscr{H}=\left\{f(x) \text { on } \mathbf{R}^{N} / \int_{\mathbf{R}^{N}}|f(x)|^{2} \mu(x) d x<\infty\right\}
$$

with its inner product

$$
(f, g)_{\mathscr{H}}=\int_{\mathbf{R}^{N}} f(x) \overline{g(x)} \mu(x) d x
$$

and norm

$$
\|f\|_{\mathscr{H}}=\left[(f, f)_{\mathscr{H}}\right]^{1 / 2} .
$$

Since $\mu(x)$ is a positive step function on $\mathbf{R}^{N}$, the Hilbert space $\mathscr{H}$ and $L_{2}\left(\mathbf{R}^{N}\right)$ are the same as sets.

Let us define the operator $H$ in $\mathscr{H}$ by

$$
\begin{aligned}
& H u=-\mu(x)^{-1} \Delta, \\
& D(H)=H^{2}\left(\mathbf{R}^{N}\right),
\end{aligned}
$$

where $D(H)$ means the domain of $H$ and the Sobolev space $H^{2}\left(\mathbf{R}^{N}\right)$ is regarded as a subset of $\mathscr{H}$. It is easy to see that $H$ is a selfadjoint operator. We denote the resolvent $(H-z)^{-1}$ by $R(z)$.

In order to evaluate some integrals over the separating surface $S$ let us prepare the following lemma due to Eidus [4].

LEMMA 2.1 (Eidus [4], (2.9)). Suppose that $\mu(x)$ is a bounded measurable function on $\mathbf{R}^{N}$ such that inf $\mu(x)>0$. Let $H$ and $R(z)$ be as above. Let

$$
u=u(\cdot, z, f)=R(z) f
$$


with $f \in L_{2}\left(\mathbf{R}^{N}\right)$ and $z=\lambda+i \eta \in \Pi$, $\Pi$ being a bounded set in $\mathbf{C} \backslash \mathbf{R}$. Then there exists a constant $C=C(\Pi, \mu)$ depending only on $\Pi$ and $\mu(x)$ such that

$$
|\eta| \int_{\mathbf{R}^{N}}\left(|\nabla u|^{2}+|u|^{2}\right) d x \leq C(|f|,|u|)_{0} .
$$

Here ( , ) 0 is the inner product of $L_{2}\left(\mathbf{R}^{N}\right)$.

Proof. Let us first notice that $u=R(z) f, z \in \mathbf{C} \backslash \mathbf{R}$, satisfies the equation

$$
-\Delta u-z \mu(x) u=\mu(x) f
$$

and $u$ belongs to $H^{2}\left(\mathbf{R}^{N}\right)$. Multiply both sides of $(2.14)$ by $\bar{u}$, integrate over $\mathbf{R}^{N}$ and take the imaginary part. Then the estimate for the term $|\eta| \int|u|^{2} d x$ is obtained. If we multiply both sides of (2.14) by $|\eta| \bar{u}$ and take the real part, we get the estimate for the term $|\eta| \int|\nabla u|^{2} d x$. $\square$

Let us now study the surface integrals of $u=R(z) f$ over the separating surface $S$. Suppose that $v \in H^{2}\left(\mathbf{R}^{N}\right)_{\text {loc }}$. Then the traces of $v$ and $\partial_{j} v(j=1,2, \ldots, N)$ on $S$ are well-defined as elements of $L_{2}(S)_{\text {loc. }}$. These traces on $S$ will be denoted as $v$ and $\partial_{j} v$ again. As usual, the inner product $(,)_{S}$ and norm \|\|$_{S}$ of $L_{2}(s)$ are defined by

$$
(v, w)_{S}=\int_{S} v \bar{w} d S \text { and }\|v\|_{S}=\left[(v, v)_{S}\right]^{1 / 2}
$$

LEMMA 2.2 (Eidus [4], (2.14)). Assume (1.6). Let $u=R(z) f$ with $f \in L_{2}\left(\mathbf{R}^{N}\right)$ and $z=\lambda+i \eta \in \mathbf{C} \backslash \mathbf{R}$. Then $u \in L_{2}(S)$ and there exists $a$ positive constant $C=C(\mu)$ depending only on $\mu(x)$ such that

$$
|\lambda|\|u\|_{S}^{2} \leq C\left(|f|,|u|+\left|\partial_{N} u\right|\right)_{0} .
$$

Here ( , ) o means the inner product of $L_{2}\left(\mathbf{R}^{N}\right)$.

Proof. Multiply both sides of (2.14) by $\partial_{N} \bar{u}$, integrate over $\mathbf{R}^{N}$ and take the real part. Then

$$
\begin{gathered}
-\operatorname{Re} \int_{\mathbf{R}^{N}}(\Delta u) \partial_{N} \bar{u} d x-\operatorname{Re} \int_{\mathbf{R}^{N}} z \mu(x) u\left(\partial_{N} \bar{u}\right) d x \\
=\operatorname{Re} \int_{\mathbf{R}^{N}} \mu(x) f\left(\partial_{N} \bar{u}\right) d x .
\end{gathered}
$$

By the use of integration by parts, we have

$$
\begin{gathered}
\operatorname{Re} \int_{\mathbf{R}^{N}}\left(\partial_{j}^{2} u\right)\left(\partial_{N} \bar{u}\right) d x=-\operatorname{Re} \int_{\mathbf{R}^{N}}\left(\partial_{j} u\right)\left(\partial_{N} \partial_{j} \bar{u}\right) d x \\
=-2^{-1} \int_{\mathbf{R}^{N}} \partial_{N}\left\{\left|\partial_{j} u\right|^{2}\right\} d x=0
\end{gathered}
$$


for $j=1,2, \ldots, N$, which implies the first term in the left-hand side of (2.17) is identically zero. Let us next estimate the second term of the left-hand side of (2.17):

$$
\begin{aligned}
-\operatorname{Re} \int_{\mathbf{R}^{N}} z & \mu(x) u\left(\partial_{N} \bar{u}\right) d x \\
= & \lambda \operatorname{Re} \int_{\mathbf{R}^{N}} \mu(x) u\left(\partial_{N} \bar{u}\right) d x \\
& \quad-\eta \operatorname{Im} \int_{\mathbf{R}^{N}} \mu(x) u\left(\partial_{N} \bar{u}\right) d x \equiv I_{1}-I_{2} .
\end{aligned}
$$

The terms $I_{1}$ and $I_{2}$ are estimated as follows:

$$
\begin{aligned}
\left|I_{1}\right| & =(|\lambda| / 2)\left|\int_{\mathbf{R}^{N}} \mu(x) \partial_{N}\left(|u|^{2}\right) d x\right| \\
& =\left.2^{-1}\left|\lambda\left(\mu_{2}-\mu_{1}\right)\right|\left|\int_{S}\right| u\right|^{2} n_{2 N}(x) d S \mid \\
& \geq 2^{-1} c_{1}\left|\lambda\left(\mu_{2}-\mu_{1}\right)\right| \int_{S}|u|^{2} d S,
\end{aligned}
$$

where we have used (1.6), and

$$
\left|I_{2}\right| \leq|\eta|\left(|u|,\left|\partial_{N} u\right|\right)_{0} \leq C_{1}(|f|,|u|)_{0}
$$

with a constant $C_{1}$, where we have used Lemma 2.1. The right-hand side of (2.17) is estimated as

$$
\left|\operatorname{Re} \int_{\mathbf{R}^{N}} \mu(x) f\left(\partial_{N} \bar{u}\right) d x\right| \leq \operatorname{Max}\left(\mu_{1}, \mu_{2}\right)\left(|f|,\left|\partial_{N} u\right|\right)_{0} .
$$

Combining these estimates, we obtain (2.16).

Using Lemmas 2.1 and 2.2, Eidus proved the following proposition:

Proposition 2.3 (Eidus [4], Lemma 2.1). Let us assume (1.6). Let $f \in L_{2}\left(\mathbf{R}^{N}\right), \gamma>3 / 2$ for $N=3, \gamma=3 / 2$ for $N>3$,

$$
0<|\eta|<1 \text { and }-1 \leq \lambda \leq M \text {, }
$$

where $z=\lambda+i \eta$ as above and $M$ is some constant. Then

$$
\|u\|_{-\gamma, 1} \leq C(|f|,|u|+|\nabla u|)_{0}
$$

for $u=R(z) f$, where the constant $C=C(M, \gamma)$ does not depend on $f, \lambda, \eta$ (but may depend on $M, \gamma)$.

For the proof see Eidus [4], p. 33-34. As for $\partial_{j} u$ on the separating surface $S$ we have the following estimates: 
Lemma 2.4. Assume (1.6). Let $u=R(z) f$ with $f \in L_{2}\left(\mathbf{R}^{N}\right)$ and $z \in \Pi$, $\Pi$ being a bounded set in $\mathbf{C} \backslash \mathbf{R}$. Then there exists a positive constant $C=C(\Pi, \mu)$ depending only on $\Pi$ and $\mu(x)$ such that

$$
\|\nabla u\|_{S} \leq C\left\{\|u\|_{0}+\|f\|_{0}\right\},
$$

where \|\|$_{0}$ is the norm of $L_{2}\left(\mathbf{R}^{N}\right)$ and

$$
\|\nabla u\|_{S}^{2}=\sum_{j=1}^{N}\left\|\partial_{j} u\right\|_{S}^{2}
$$

Proof. Let $B_{R}=\left\{x \in \mathbf{R}^{N} /|x|<R\right\}$ and $S_{R}=\left\{x \in \mathbf{R}^{N} /|x|=R\right\}$ for $R>0$. By the Green formula we have

$$
\begin{aligned}
\int_{\Omega_{1} \cap B_{R}} & \partial_{N}\left(|\nabla u|^{2}\right) d x \\
= & \int_{S \cap B_{R}}|\nabla u|^{2} n_{1 N}(x) d S+\int_{\Omega_{1} \cap S_{R}}|\nabla u|^{2} \tilde{x}_{n} d S,
\end{aligned}
$$

where $n_{1 N}(x)$ is the $N$ th element of the outward normal of $\partial \Omega_{1}$ at $x$ and $\tilde{x}_{N}=x_{N} /|x|$. By using (1.6) we obtain from (2.27)

$$
\begin{aligned}
& c \int_{S \cap B_{R}}|\nabla u|^{2} d S \\
& \quad \leq 2 \sum_{j=1}^{N} \int_{\Omega_{1} \cap B_{R}}\left|\partial_{N} \partial_{j} u\right|\left|\partial_{j} u\right| d x+\int_{\Omega_{1} \cap S_{R}}|\nabla u|^{2} d S .
\end{aligned}
$$

Let $R \rightarrow \infty$ along an appropriate sequence $\left\{R_{m}\right\}$ so that the second term of the right-hand side of $(2.28)$ converges to zero. Then we have

$$
\int_{S}|\nabla u|^{2} d S \leq C_{2}\|u\|_{2,0}^{2}
$$

with a constant $C_{2}$, where \|\|$_{2,0}$ is the norm of $H^{2}\left(\mathbf{R}^{N}\right)$. The estimate (2.25) follows from (2.29), the well-known estimate

$$
\|u\|_{2,0} \leq C_{3}\left\{\|\Delta u\|_{0}+\|u\|_{0}\right\}
$$

with a constant $C_{3}$ and the equation (2.14).

3. More a priori estimates. In this section we shall introduce a "modified radiation condition-like" term for the solution $u$ of the equation (2.14), i.e., $-\Delta u-z \mu(x) u=\mu(x) f$, where $z \in \mathbf{C} \backslash \mathbf{R}$. Some estimates for it will be proved. These estimates can be regarded as modifications of the estimates of the usual radiation conditions given in, e.g., [6], [5], [11], although we treat here only the case that $z \in \mathbf{C} \backslash \mathbf{R}$. 
By using these estimates, we shall also show some estimates for the norm $\|u\|_{-\tau}$ with $\tau>1 / 2$. They will be used in $\S 4$ to show the limiting absorption principle for the operator $H$.

Let us start with some notation. For $z \in \mathbf{C} \backslash \mathbf{R}$ and $x \in \mathbf{R}^{N}$ we set

$$
\begin{aligned}
& k=k(x)=k(x, z)=[z \mu(x)]^{1 / 2}, \\
& a=a(x)=a(x, z)=\operatorname{Re} k(x, z), \\
& b=b(x)=b(x, z)=\operatorname{Im} k(x, z),
\end{aligned}
$$

where the branch of $[z \mu(x)]^{1 / 2}$ is taken so that $b(x, z)>0$. With fixed $z$ the functions $k(x), a(x)$ and $b(x)$ are step functions on $\mathbf{R}^{N}$ which are constant in each $\Omega_{1}$ and $\Omega_{2}$. Let us next introduce some differential expressions of the first order;

$$
\begin{array}{r}
\mathscr{D}_{j} u=\partial_{j} u+\{(N-1) /(2 r)\} \tilde{x}_{j} u-i k(x) \tilde{x}_{j} u \\
\left(r=|x|, \tilde{x}_{j}=x_{j} / r, j=1,2, \ldots, N\right),
\end{array}
$$

$$
\begin{aligned}
\mathscr{D} u=\nabla u+\{(N-1) /(2 r)\} \tilde{x} u-i k(x) \tilde{x} u \\
\\
\left(\tilde{x}=\left(\tilde{x}_{1}, \tilde{x}_{2}, \ldots, \tilde{x}_{N}\right)\right),
\end{aligned}
$$

$$
|\mathscr{D} u|=\left[\sum_{j=1}^{N}\left|\mathscr{D}_{j} u\right|^{2}\right]^{1 / 2}
$$

$$
\mathscr{D}_{r} u=\mathscr{D} u \cdot \tilde{x}=\frac{\partial u}{\partial r}+\{(N-1) /(2 r)\} u-i k(x) u
$$

(3.8) $\mathscr{D}_{n} u=\mathscr{D} u \cdot n=\frac{\partial u}{\partial n}+\{(N-1) /(2 r)\}(\tilde{x} \cdot n) u-i k(x)(\tilde{x} \cdot n) u$, where $n$ is a unit vector in $\mathbf{R}^{N}$.

LEMMA 3.1. Let $u \in H^{2}\left(\mathbf{R}^{N}\right)_{\text {loc }}$ and set $f=\mu(x)^{-1}\left(-\Delta-k^{2}\right) u$, where $k=[z \mu(x)]^{1 / 2}$ with $z \in \mathbf{C} \backslash R$. Let $\xi \in C^{1}([0, \infty))$ with $\xi(r)=0$ 
in a neighborhood of $r=0$ and set $\varphi(x)=\xi(|x|)$. Then we have

$$
\text { (3.9) } \begin{aligned}
2^{-1} \int_{B_{R}} & \frac{\partial \varphi}{\partial r}|\mathscr{D} u|^{2} d x+\sum_{l=1}^{2} \int_{\partial \Omega_{l} \cap B_{R}} \varphi \cdot \operatorname{Im}\left\{\bar{k} \cdot \frac{\partial u}{\partial n} \cdot \bar{u}\right\} d S \\
& +\int_{B_{R}} b \varphi|\mathscr{D} u|^{2} d x \\
& +\int_{B_{R}}\left(\frac{\varphi}{r}-\frac{\partial \varphi}{\partial r}\right)\left(|\mathscr{D} u|^{2}-\left|\mathscr{D}_{r} u\right|^{2}\right) d x \\
& +c_{N} \int_{B_{R}} r^{-2}\left(\frac{\varphi}{r}-2^{-1} \frac{\partial \varphi}{\partial r}+b \varphi\right)|u|^{2} d x \\
= & \operatorname{Re} \int_{B_{R}} \varphi \mu(x) f \overline{\left(\mathscr{D}_{r} u\right)} d x \\
& \left.+2^{-1} \sum_{l=1}^{2} \int_{\partial \Omega_{l} \cap B_{R}} \varphi \frac{(N-1) b}{r}+|k|^{2}\right\}(\tilde{x} \cdot n)|u|^{2} d x \\
& +2^{-1} \int_{S_{R}} \varphi\left(2\left|\mathscr{D}_{r} u\right|^{2}-|\mathscr{D} u|^{2}-c_{N} r^{-2}|u|^{2}\right) d x
\end{aligned}
$$

for each $R>0$, where $\partial / \partial n$ in the integrand of the surface integral over $\partial \Omega_{l} \cap B_{R}$ means the directional derivative in the directon of the outward normal of $\partial \Omega_{l}, B_{R}$ is the open ball with center $x=0$ and radius $R, S_{R}$ is the sphere with center $x=0$ and radius $R$ and

$$
c_{N}=(N-1)(N-3) / 4 \text {. }
$$

Proof. Since $k(x)$ is a constant $\left[z \mu_{1}\right]^{1 / 2}$ or $\left[z \mu_{2}\right]^{1 / 2}$ in each region $\Omega_{1}$ or $\Omega_{2}$, the equation $-\Delta u-k^{2} u=\mu(x) f$ is rewritten as

$$
-\sum_{j=1}^{N} \partial_{j} \mathscr{D}_{j} u+\left\{\frac{N-1}{2 r}-i k\right\} \mathscr{D}_{r} u+V_{0}(x) u=\mu(x) f
$$

in $\Omega_{1}$ or $\Omega_{2}$, where $\mathscr{D}_{j} u$ and $\mathscr{D}_{r} u$ are as above, $\partial_{j}=\partial / \partial x_{j}$ and $V_{0}(x)$ is given by

$$
V_{0}(x)=c_{N} r^{-2}
$$

(cf. Ikebe \& Saitō [5], (2.10)). Multiply the both sides of (3.11) by $\varphi \overline{\left(\mathscr{D}_{r} u\right)}$, integrate on each $B_{R} \cap \Omega_{l}(l=1,2)$ and take the real part. Then, using integration by parts or the Green formula and making the sum of these two integrals, we obtain the relation (3.9) (cf. [5], Lemma 2.2).

In order to get our first a priori estimate we are going to introduce the following weight functions. 
Notation 3.2. (i) Let $\rho(r)$ be a $C^{2}$ function on $[0, \infty)$ such that

$$
\rho(r)=0 \quad\left(0 \leq r \leq R_{0}\right), \quad=1 \quad\left(r \geq R_{0}+1\right)
$$

with $R_{0}>0,0 \leq \rho(r) \leq 1$ and $\rho^{\prime}(r) \geq 0$.

(ii) For each $\varepsilon>0$ the function $\sigma_{\varepsilon}(r)$ is defined by

$$
\sigma_{\varepsilon}(r)=\exp \left\{-\varepsilon^{-1}(1+r)^{-\varepsilon}\right\} \quad(r \geq 0) .
$$

Proposition 3.3. Let us assume Assumption 1.1. Let $u=R(z) f$ with $z=\lambda+i \eta \in \mathbf{C} \backslash \mathbf{R}$ and $f \in L_{2}\left(\mathbf{R}^{N}\right)$. Here $z=\lambda+$ in moves in $a$ bounded set $K$ of $\mathbf{C} \backslash \mathbf{R}$ such that

$$
|\lambda| \geq \lambda_{0}>0 \quad(z=\lambda+i \eta \in K)
$$

with a positive number $\lambda_{0}$. Then there exists a constant $C=C(K)$ depending only on $K$ such that

$$
\begin{aligned}
2^{-1} \int_{\mathbf{R}^{N}} \frac{\partial}{\partial r}\left\{\rho^{2} \sigma_{\varepsilon}\right\}|\mathscr{D} u|^{2} d x & \\
& +\sum_{l=1}^{2} \int_{\partial \Omega_{l}} \rho^{2} \sigma_{\varepsilon} \cdot \operatorname{Im}\left\{\bar{k} \cdot \frac{\partial u}{\partial n} \cdot \bar{u}\right\} d S \\
\leq C & \left\{\left(\sigma_{\varepsilon}|f|,|\nabla u|+|u|\right)_{0, E_{R_{0}}}\right. \\
& \left.+\int_{E_{R_{0}}}(1+|x|)^{-2} \sigma_{\varepsilon}\left(|\nabla u|^{2}+|u|^{2}\right) d x\right\}
\end{aligned}
$$

holds for each $0<\varepsilon$, where

$$
E_{R_{0}}=\left\{x /|x|>R_{0}\right\}
$$

the functions $\rho$ and $\sigma_{\varepsilon}$ are as in Notation 3.2 with $r=|x|$.

Proof. Set $\varphi(x)=\rho(|x|)^{2} \sigma_{\varepsilon}(|x|)$ in (3.9). We are going to evaluate each term of (3.9). Noting that

$$
\frac{\varphi}{r}-\frac{\partial \varphi}{\partial r}=\rho^{2} \sigma_{\varepsilon}\left\{r^{-1}-(1+r)^{-1-\varepsilon}\right\}-2 \rho \rho^{\prime} \sigma_{\varepsilon} \geq-2 \rho \rho^{\prime} \sigma_{\varepsilon}
$$

and

$$
|\mathscr{D} u|^{2}-\left|\mathscr{D}_{r} u\right|^{2}=|\nabla u|^{2}-\left|\frac{\partial u}{\partial r}\right|^{2} \geq 0
$$


we have for $R>R_{0}$

$$
\begin{aligned}
\int_{B_{R}} & \left\{\frac{\varphi}{r}-\frac{\partial \varphi}{\partial r}\right\}\left\{|\mathscr{D} u|^{2}-\left|\mathscr{D}_{r} u\right|^{2}\right\} d x \\
& \geq-2 \int_{B_{R}} \rho \rho^{\prime} \sigma_{\varepsilon}\left\{|\nabla u|^{2}-|u|^{2}\right\} d x \geq-2 \int_{B_{R}} \rho \rho^{\prime} \sigma_{\varepsilon}|\nabla u|^{2} d x .
\end{aligned}
$$

Therefore it follows from (3.9) that

$$
\begin{aligned}
2^{-1} & \int_{B_{R}} \frac{\partial \varphi}{\partial r}|\mathscr{D} u|^{2} d x+\sum_{l=1}^{2} \int_{\partial \Omega_{l} \cap B_{R}} \varphi \cdot \operatorname{Im}\left\{\bar{k} \cdot \frac{\partial u}{\partial n} \cdot \bar{u}\right\} d S \\
\leq & \operatorname{Re} \int_{B_{R}} \varphi \cdot \mu \cdot f \cdot \overline{\left(\mathscr{D}_{r} u\right)} d x+2 \int_{B_{R}} \rho \rho^{\prime} \sigma_{\varepsilon}|\nabla u|^{2} d x \\
& +2^{-1} \sum_{l=1}^{2} \int_{\partial \Omega_{l} \cap B_{R}} \varphi\left\{\frac{(N-1) b}{r}+|k|^{2}\right\}(\tilde{x} \cdot n)|u|^{2} d S \\
& -c_{N} \int_{B_{R}} r^{-2}\left(\frac{\varphi}{r}-2^{-1} \frac{\partial \varphi}{\partial r}+b \varphi\right)|u|^{2} d x \\
& +2^{-1} \int_{S_{R}} \varphi\left(2\left|\mathscr{D}_{r} u\right|^{2}-|\mathscr{D} u|^{2}-c_{N} r^{-2}|u|^{2}\right) d x .
\end{aligned}
$$

Since $u=R(z) f \in H^{2}\left(\mathbf{R}^{N}\right)$, the fifth term of the right-hand side of (3.21) will converge to zero when $R \rightarrow \infty$ along a suitable sequence $\left\{R_{m}\right\}$. Thus we obtain from (3.21)

$$
\begin{aligned}
2^{-1} \int_{\mathbf{R}^{N}} & \frac{\partial \varphi}{\partial r}|\mathscr{D} u|^{2} d x+\sum_{l=1}^{2} \int_{\partial \Omega_{l}} \varphi \cdot \operatorname{Im}\left\{\bar{k} \cdot \frac{\partial u}{\partial n} \cdot \bar{u}\right\} d S \\
\leq & \operatorname{Re} \int_{\mathbf{R}^{N}} \varphi \cdot \mu \cdot f \cdot \overline{\left(\mathscr{D}_{r} u\right)} d x+2 \int_{\mathbf{R}^{N}} \rho \rho^{\prime} \sigma_{\varepsilon}|\nabla u|^{2} d x \\
& +2^{-1} \sum_{l=1}^{2} \int_{\partial \Omega_{l}} \varphi\left\{\frac{(N-1) b}{r}+|k|^{2}\right\}(\tilde{x} \cdot n)|u|^{2} d S \\
& +c_{N} \int_{\mathbf{R}^{N}} r^{-2}\left(\frac{\varphi}{r}-2^{-1} \frac{\partial \varphi}{\partial r}+b \varphi\right)|u|^{2} d x,
\end{aligned}
$$

where we should note that the surface integrals over $\partial \Omega_{l}$ in (3.22) is absolutely convergent, because $u, \nabla u \in L_{2}(S)$ by Lemmas 2.2 and 2.4.

Let us denote by $J$ the third term of the right-hand side of (3.22) and let us estimate the surface integral $J$. It follows from (1.7) in Assumption 1.1 that

$$
|J| \leq C_{1} \int_{S} \varphi(1+|x|)^{-1}|u|^{2} d S \quad(z \in K),
$$


where the constant $C_{1}=C_{1}(K)$ depends only on the bounded set $K$ (and the constant $c_{2}$ in (1.7)). Set

$$
v(x)=\varphi^{1 / 2}(1+r)^{-1 / 2} u=\rho \sigma_{\varepsilon}^{1 / 2}(1+r)^{-1 / 2} u \equiv \psi u .
$$

Since $v$ satisfies the equation

$$
-\Delta v-k^{2} v=\psi \mu f-2(\nabla \psi) \cdot(\nabla u)-(\Delta \psi) u \equiv F
$$

it follows that Lemma 2.2 that

$$
|J| \leq C_{1} \int_{S}|v|^{2} d S \leq C_{2}\left(|F|,\left|\psi u+\partial_{N}(\psi u)\right|\right)_{0}
$$

with a constant $C_{2}=C_{2}(K)$. By a straightforward computation we get (3.27)

$$
\begin{aligned}
&\left|\partial^{\alpha} \psi(x)\right| \leq C_{3} \sigma_{\varepsilon}(|x|)^{1 / 2}(1+|x|)^{-(1 / 2)-|\alpha|} \\
&\left(|x| \geq R_{0},|\alpha|=0,1,2\right),
\end{aligned}
$$

with $\partial^{\alpha} \psi(x)=0$ for $|x| \leq R_{0}(|\alpha|=0,1,2)$, where $C_{3}$ is a positive constant depending only on $R_{0}$ though the constant $R_{0}$ is fixed throughout this work. Since it follows from (3.27) that

(3.28) $|F| \cdot\left|\psi u+\partial_{N}(\psi u)\right| \leq C_{4} \sigma_{\varepsilon}\{|f| \cdot(|\nabla u|+|u|)$

$$
\left.+(1+|x|)^{-2}\left(|\nabla u|^{2}+|u|^{2}\right)\right\}
$$

with a constant $C_{4}=C_{4}(K)$, we have

$$
\begin{aligned}
|J| \leq C_{5}\{ & \left(\sigma_{\varepsilon}|f|,|\nabla u|+|u|\right)_{0, E_{R_{0}}} \\
& \left.+\int_{E_{R_{0}}}(1+|x|)^{-2} \sigma_{\varepsilon}\left(|\nabla u|^{2}+|u|^{2}\right) d x\right\}
\end{aligned}
$$

with $C_{5}=C_{5}(K)$.

The other three terms in the right-hand side of (3.22) can be easily evaluated. Thus we get (3.16).

Corollary 3.4. Let $p, \sigma_{\varepsilon}, K$ and $\varphi=\rho^{2} \sigma_{\varepsilon}$ be as above. Then there exists a positive constant $\tilde{C}=\tilde{C}(K)$ such that

$$
\begin{aligned}
& \text { (3.30) } \int_{E_{R_{0}}} \frac{\partial \varphi}{\partial r}|\nabla u-i k \tilde{x} u|^{2} d x+2 \sum_{l=1}^{2} \int_{\partial \Omega_{l}} \varphi \cdot \operatorname{Im}\left\{\bar{k} \cdot \frac{\partial u}{\partial n} \cdot \bar{u}\right\} d S \\
& \leq \tilde{C}\left\{\left(\sigma_{\varepsilon}|f|,|\nabla u|+|u|\right)_{0, E_{R_{0}}}\right. \\
& \left.+\int_{R_{0}}(1+|x|)^{-2} \sigma_{\varepsilon}\left(|\nabla u|^{2}+|u|^{2}\right) d x\right\}
\end{aligned}
$$

holds for $u=R(z) f$ with $f \in L_{2}\left(\mathbf{R}^{N}\right), \varepsilon>0$ and $z \in K$. 
Proof. The estimate (3.30) directly follows from (3.16) by noting that

(3.31) $|\mathscr{D} u|^{2}-|\nabla u-i k \tilde{x} u|^{2}$

$$
=\frac{N-1}{2 r} \cdot\left(\frac{\partial|u|^{2}}{\partial r}\right)-\frac{(N-1) b|u|^{2}}{r}+\frac{(N-1)^{2}|u|^{2}}{4 r^{2}} .
$$

REMARK 3.5. (i) It should be noted that the constants $C$ in (3.16) and $\tilde{C}$ in (3.30) do not depend on $\varepsilon$ in the function $\sigma_{\varepsilon}$.

(ii) The estimate (3.16) or (3.30) can be regarded as a (weaker) generalization of the estimate

$$
\int_{\mathbf{R}^{N}}(1+|x|)^{2 \delta-2}|\nabla v-i k \tilde{x} v|^{2} d x \leq C\left\{\|g\|_{\delta}^{2}+\|v\|_{-\delta}^{2}\right\}
$$

with $\delta>1 / 2$ for solutions $v$ of the Schrödinger equation

$$
\left(-\Delta+Q(x)-k^{2}\right) v=g
$$

with a short-range or long-range potential (cf. e.g., Ikebe \& Saitō [5], Lemma 1.7).

The estimate obtained above will be used to prove an a priori estimate of the $H_{-\delta}^{1}\left(\mathbf{R}^{N}\right)$-norm of $u=R(z) f$. First we need the following lemma.

LEMMA 3.6. Let $S$ be the separting surface as above. Then there exists $R_{1}>0$ such that

$$
\begin{array}{rl}
\int_{S \cap E_{R}} & F(x) d S \\
& =\int_{R}^{\infty}\left\{\int_{S^{N-2}} F(t \omega, \varphi(t \omega)) \frac{\left(1+|\nabla \varphi|^{2}\right)^{1 / 2} t^{N-2} r}{t+\varphi(t \omega) \cdot(\nabla \varphi \cdot \omega)} d \omega\right\} d r
\end{array}
$$

holds for any $R \geq R_{1}$ and any integrable function $F(x)$ over $S \cap E_{R}$, where $E_{R}=\left\{x \in \mathbf{R}^{N} /|x|>R\right\}, s^{N-2}$ is the unit sphere in $\mathbf{R}^{N-1}, \omega \in$ $S^{N-2}, t=t(r, \omega)$ is defined by $r=\left(t^{2}+\varphi(t \omega)^{2}\right)^{1 / 2}$ and $\nabla \varphi=(\nabla \varphi)(t \omega)$.

Proof. By use of the relation

$$
\begin{aligned}
d S & =\left(1+|\nabla \varphi|^{2}\right)^{1 / 2} d x^{\prime} \\
& =\left(1+|\nabla \varphi|^{2}\right)^{1 / 2} t^{N-2} d t d \omega \quad\left(x^{\prime}=t \omega\right)
\end{aligned}
$$

it follows that

$$
\begin{aligned}
& \int_{S \cap E_{R}} F d S \\
& =\int_{S^{N-2}} d \omega \int_{\left(t^{2}+\varphi(t \omega)^{2}\right)^{1 / 2}>R} F(t \omega, \varphi(t \omega))\left(1+|\nabla \varphi|^{2}\right)^{1 / 2} t^{N-2} d t
\end{aligned}
$$


For fixed $\omega \in S^{N-2}$ let us consider the relation

$$
r=\left(t^{2}+\varphi(t \omega)^{2}\right)^{1 / 2}
$$

Then

$$
\frac{d r}{d t}=\left(t+\varphi(t \omega) \cdot \frac{\partial \varphi(t \omega)}{\partial t}\right)\left(t^{2}+\varphi(t \omega)^{2}\right)^{-1 / 2}
$$

Since the outward normals $n_{l}(x)$ of $\partial \Omega_{l}$ are expressed as

$$
\begin{array}{r}
n_{l}(x)= \pm\left(1+\left|\nabla \varphi\left(x^{\prime}\right)\right|^{2}\right)^{-1 / 2}\left(\frac{\partial \varphi}{\partial x_{1}}, \frac{\partial \varphi}{\partial x_{2}}, \ldots, \frac{\partial \varphi}{\partial x_{N-1}},-1\right) \\
\left(x=\left(x^{\prime}, x_{N}\right) \in S\right)
\end{array}
$$

the inner product $n_{l}(x) \cdot x(x \in S)$ in $\mathbf{R}^{N}$ has the form

$$
n_{l}(x) \cdot x= \pm\left(1+\left|\nabla \varphi\left(x^{\prime}\right)\right|^{2}\right)^{-1 / 2}\left\{r^{\prime} \frac{\partial \varphi\left(x^{\prime}\right)}{\partial r^{\prime}}-\varphi\left(x^{\prime}\right)\right\}
$$

with $r^{\prime}=\left|x^{\prime}\right|$. By setting $x^{\prime}=t \omega$ in (3.39) and using (1.6) and (1.7) in Assumption 1.1, we obtain

$$
\frac{\partial \varphi(t \omega)}{\partial t}=t^{-1} \varphi(t \omega)+O\left(t^{-1}\right) \quad(t \rightarrow \infty)
$$

where the term $O\left(t^{-1}\right)$ is uniform for $\omega \in S^{N-2}$. Here we should note that the boundedness of $\left|\nabla \varphi\left(x^{\prime}\right)\right|$ on $\mathbf{R}^{N-1} \backslash\{0\}$ follows from (1.6) since the $N$ th component $n_{l N}(x)$ of the outward normal $n_{l}(x)$ has the form $\pm\left(1+\left|\nabla \varphi\left(x^{\prime}\right)\right|\right)^{-1 / 2}$. It follows from (3.40) that

$$
\begin{aligned}
& \frac{d r}{d t}=\left\{t+t^{-1} \varphi(t \omega)^{2}+\varphi(t \omega) \cdot O\left(t^{-1}\right)\right\}\left\{t^{2}+\varphi(t \omega)^{2}\right\}^{-1 / 2} \\
& \left.=\left\{1+t^{-2} \varphi(t \omega)^{2}+t^{-1} \varphi(t \omega) \cdot O\left(t^{-1}\right)\right\}\left\{1+t^{-2} \varphi(t \omega)^{2}\right)\right\}^{-1 / 2} \\
& =\left\{1+t^{-2} \varphi(t \omega)^{2}\right\}^{1 / 2}+t^{-1} \varphi(t \omega)\left\{1+t^{-2} \varphi(t \omega)^{2}\right\}^{-1 / 2} \cdot O\left(t^{-1}\right) \\
& =\left\{1+t^{-2} \varphi(t \omega)^{2}\right\}^{1 / 2}+O\left(t^{-1}\right)
\end{aligned}
$$

as $t \rightarrow \infty$. Therefore there exists a positive number $R_{1}\left(\geq R_{0}\right)$ such that $d r / d t>0$ for all $r \geq R_{1}$. Therefore the inverse function $t=t(r)=$ $t(r, \omega)$ is well-defined and is a $C^{1}$ function. Thus we have (3.33).

In the following proposition we shall study some integrals which are closely related to the left-hand side of (3.30).

Proposition 3.7. Let us assume Assumption 1.1. Let $u=R(z) f$ with $z \in \mathbf{C} \backslash \mathbf{R}$ and $f \in L_{2}\left(\mathbf{R}^{N}\right)$. 
(i) Then we have

$$
\begin{aligned}
& \sum_{l=1}^{2} \int_{\partial \Omega_{l}} \operatorname{Im}\left\{\bar{k} \cdot \frac{\partial u}{\partial n} \cdot \bar{u}\right\} d S \\
& =-\int_{\mathbf{R}^{N}} b\left(|\nabla u|^{2}+|k|^{2}|u|^{2}\right) d x \\
& \quad-\int_{\mathbf{R}^{N}} \mu(x) \cdot \operatorname{Im}(\bar{k} \cdot f \cdot \bar{u}) d x,
\end{aligned}
$$

where $k=[z \mu]^{1 / 2}, b=\operatorname{Im} k$ and $n$ in the surface integral over $\partial \Omega_{l}$ means the outward unit normal of $\partial \Omega_{l}$.

(ii) We have

$$
\begin{aligned}
\int_{S_{r}} & |\nabla u-i k \tilde{x} u|^{2} d S+2 \sum_{l=1}^{2} \int_{\partial \Omega_{l} \cap E_{r}} \operatorname{Im}\left\{\bar{k} \cdot \frac{\partial u}{\partial n} \cdot \bar{u}\right\} d S \\
= & \int_{S_{r}}\left(|\nabla u|^{2}+|k|^{2}|u|^{2}\right) d S-2 \int_{E_{r}} b\left(|\nabla u|^{2}+|k|^{2}|u|^{2}\right) d x \\
& -2 \int_{E_{r}} \mu(x) \cdot \operatorname{Im}\{\bar{k} \cdot f \cdot \bar{u}\} d x,
\end{aligned}
$$

for $r>0$, where $S_{r}=\left\{x \in \mathbf{R}_{N} /|x|=r\right\}, E_{r}=\left\{x \in \mathbf{R}^{N} /|x|>r\right\}$ and $n$ in the surface integral over $\partial \Omega_{l} \cap E_{r}$ means the outward unit normal of $\partial \Omega_{l}$.

Proof. Multiply the equation $-\Delta u-k^{2} u=\mu f$ by $\overline{k u}$ and integrate on $E_{r}$. Then we have

$$
\begin{aligned}
(3.44)-\sum_{l=1}^{2} & \int_{\partial \Omega_{l} \cap E_{r}} \bar{k} \cdot \frac{\partial u}{\partial n} \cdot \bar{u} d S+\int_{S_{r}} \bar{k} \cdot \frac{\partial u}{\partial r} \cdot \bar{u} d S \\
& +\int_{E_{r}} \bar{k}|\nabla u|^{2} d x-\int_{E_{r}} k|k|^{2}|u|^{2} d x=\int_{E_{r}} \bar{k} \mu(x) f \bar{u} d x .
\end{aligned}
$$

The relation (3.42) is obtained by taking the imaginary part of (3.44) and letting $r \rightarrow 0$ along a suitable sequence $\left\{r_{m}\right\}$. The second relation (3.43) follows from (3.44) and

$$
\begin{aligned}
& \int_{S_{r}}|\nabla u-i k \tilde{x} u|^{2} d S \\
& \quad=\int_{S_{r}}\left(|\nabla u|^{2}+|k|^{2}|u|^{2}\right) d S-2 \int_{S_{r}} \operatorname{Im}\left\{\bar{k} \cdot \frac{\partial u}{\partial r} \cdot \bar{u}\right\} d S
\end{aligned}
$$

Let us now evaluate the norm $\|u\|_{-\delta, E_{r}}$. 
Proposition 3.8. Suppose that Assumption 1.1 is satisfied. Let $R_{1}$ be as in Lemma 3.6. Let $\sigma_{\varepsilon}(r)$ be as in Notation 3.2. Let $u=R(z) f$ with $z \in K$ and $f \in L_{2}\left(\mathbf{R}^{N}\right)$, where $K$ is as in Proposition 3.3. Then there exists a positive constant $C=C(K)$ such that

$$
\begin{aligned}
& \int_{E_{R}}(1+|x|)^{-2 \tau} \sigma_{\varepsilon}\left(|\nabla u|^{2}+|u|^{2}\right) d x \\
& \leq C(1+R)^{-(2 \tau-1-\varepsilon)}\left\{(|f|,|\nabla u|+|u|)_{0}\right. \\
& \left.\quad+\int_{E_{R_{1}}}(1+|x|)^{-2} \sigma_{\varepsilon}\left(|\nabla u|^{2}+|u|^{2}\right) d x\right\}
\end{aligned}
$$

holds for all $R \geq R_{1}+1$ and all pairs $(\tau, \varepsilon)$ satisfying

$$
0<\varepsilon \leq 2 \tau-1
$$

where (, ) $)_{0}$ means the inner product of $L_{2}\left(\mathbf{R}^{N}\right)$.

Proof. The proof will be divided into several steps.

(I) Set

$$
\mathscr{A}(r)=\mathscr{A}(r, z, f)=\int_{S_{r}}\left(|\nabla u|^{2}+|k|^{2}|u|^{2}\right) d S
$$

Let $\rho(r)$ be as in Notation 3.2 with $R_{0}$ replaced by $R_{1}$ and let $R \geq$ $R_{1}+1$. Then we have

$$
\begin{aligned}
& \int_{E_{R}}(1+|x|)^{-2 \tau} \sigma_{\varepsilon}\left(|\nabla u|^{2}+|k|^{2}|u|^{2}\right) d x \\
& =\int_{R}^{\infty}(1+r)^{-2 \tau} \sigma_{\varepsilon}(r) \mathscr{A}(r) d r \\
& \leq(1+R)^{-(2 \tau-1-\varepsilon)} \int_{R}^{\infty}(1+r)^{-(1+\varepsilon)} \sigma_{\varepsilon}(r) \mathscr{A}(r) d r \\
& \leq(1+R)^{-(2 \tau-1-\varepsilon)} \int_{R_{1}} \rho(r)^{2}(1+r)^{-(1+\varepsilon)} \sigma_{\varepsilon}(r) \mathscr{A}(r) d r \\
& \leq(1+R)^{-(2 \tau-1-\varepsilon)} \int_{R_{1}}^{\infty} \frac{\partial}{\partial r}\left\{\rho^{2} \sigma_{\varepsilon}\right\} \mathscr{A}(r) d r,
\end{aligned}
$$

where we should note that $2 \tau-1-\varepsilon \geq 0, \rho(r)=1$ for $r \geq R_{1}+1$ and

$$
\frac{\partial}{\partial r}\left\{\rho(r)^{2} \sigma_{\varepsilon}(r)\right\} \geq \rho(r)^{2}(1+r)^{-(1+\varepsilon)} \sigma_{\varepsilon}(r) \geq 0
$$

for $r \geq R_{1}$. 
(II) It follows from (3.43) that

$$
\begin{aligned}
& \int_{R_{1}}^{\infty} \frac{\partial \varphi}{\partial r} \mathscr{A}(r) d r \\
& =\int_{R_{1}}^{\infty} \frac{\partial \varphi}{\partial r}\left[\int_{S_{r}}|\nabla u-i k \tilde{x} u|^{2} d S\right. \\
& +2 \sum_{l=1}^{2} \int_{\partial \Omega_{l} \cap E_{r}} \operatorname{Im}\left\{\bar{k} \cdot \frac{\partial u}{\partial n} \cdot \bar{u}\right\} d S \\
& +2 \int_{E_{r}} b\left(|\nabla u|^{2}+|k|^{2}|u|^{2}\right) d x \\
& \left.\quad+2 \int_{E_{r}} \mu(x) \cdot \operatorname{Im}\{\bar{k} \cdot f \cdot \bar{u}\} d x\right] d r
\end{aligned}
$$

where we set $\varphi=\rho^{2} \sigma_{\varepsilon}$. Let us first look at the term

$$
J_{l}=\int_{R_{1}}^{\infty} \frac{\partial \varphi}{\partial r}\left[\int_{\partial \Omega_{l} \cap E_{r}} \operatorname{Im}\left\{\bar{k} \cdot \frac{\partial u}{\partial n} \cdot \bar{u}\right\} d S\right] d r .
$$

It follows from Lemma 3.6 that the term $J_{l}$ is expressed in the form

$$
J_{l}=\int_{R_{1}}^{\infty} \frac{\partial \varphi}{\partial r}\left[\int_{r}^{\infty} F_{l}(s) d s\right] d r
$$

whence we get

$$
J_{l}=\int_{R_{1}}^{\infty} \varphi(r) F_{l}(r) d r=\int_{\partial \Omega_{l}} \varphi \cdot \operatorname{Im}\left\{\bar{k} \cdot \frac{\partial u}{\partial n} \cdot \bar{u}\right\} d S .
$$

Here we should note that $\varphi(r)=\rho(r)^{2} \sigma_{\varepsilon}(r)=0$ for $r \leq R_{1}$. Thus, together with Corollary 3.4 , we obtain

$$
\begin{aligned}
\int_{R_{1}}^{\infty} \frac{\partial \varphi}{\partial r}\left[\int_{S_{r}}|\nabla u-i k \tilde{x} u|^{2} d S\right. & \left.+2 \sum_{l=1}^{2} \int_{\partial \Omega_{l} \cap E_{r}} \operatorname{Im}\left\{\bar{k} \cdot \frac{\partial u}{\partial n} \cdot \bar{u}\right\} d S\right] d r \\
= & \int_{E_{R_{1}}} \frac{\partial \varphi}{\partial r}|\nabla u-i k \tilde{x} u|^{2} d x \\
& +2 \sum_{l=1}^{2} \int_{\partial \Omega_{l} \cap E_{R_{1}}} \varphi \cdot \operatorname{Im}\left\{\bar{k} \cdot \frac{\partial u}{\partial n} \cdot \bar{u}\right\} d S \\
\leq & C_{1}\left\{\left(\sigma_{\varepsilon}|f|,|\nabla u|+|u|\right)_{0, E_{R_{1}}}\right. \\
& \left.\quad+\int_{E_{R_{1}}}(1+|x|)^{-2} \sigma_{\varepsilon}\left(|\nabla u|^{2}+|u|^{2}\right) d x\right\}
\end{aligned}
$$

with a constant $C_{1}=C_{1}(K)$. 
(III) It is seen from Lemma 2.1 that we have

$$
\int_{E_{t}} b\left(|\nabla u|^{2}+|k|^{2}|u|^{2}\right) d x \leq C_{2}(|f|,|u|)_{0}
$$

with a constant $C_{2}=C_{2}(K)$, where we should note that $2 a b=\mu(x) \eta$ and $|a| \geq \sqrt{\lambda_{0}}$. Therefore we obtain

$$
\begin{gathered}
\int_{R_{1}}^{\infty} \frac{\partial \varphi}{\partial t}\left[\int _ { E _ { l } } b \left(|\nabla u|^{2}\right.\right. \\
\left.\left.+|k|^{2}|u|^{2}\right) d x+\int_{E_{t}} \mu \cdot \operatorname{Im}\{\bar{k} \cdot f \cdot \bar{u}\} d S\right] d t \\
\leq C_{3}(|f|,|u|)_{0}
\end{gathered}
$$

with $C_{3}=C_{3}(K)$, where we have used the fact that $\varphi(r) \rightarrow 1$ as $r \rightarrow \infty$. The estimate (3.46) follows from (3.49), (3.51), (3.55) and (3.57).

The estimate (3.46) can be improved in the following way.

CoRollary 3.9. Let $K, R_{1}$ be as above. Then there exist positive constants $\tilde{C}=\tilde{C}(K)$ and $R_{2}=R_{2}(K)$ such that

$$
\begin{aligned}
& \int_{E_{R}}(1+|x|)^{-2 \tau} \sigma_{\varepsilon}\left(|\nabla u|^{2}+|u|^{2}\right) d x \\
& \leq \tilde{C}(1+R)^{-(2 \tau-1-\varepsilon)}\left\{(|f|,|\nabla u|+|u|)_{0}\right. \\
& \left.\quad+\int_{B_{R_{1}, R_{2}}}(1+|x|)^{-2} \sigma_{\varepsilon}\left(|\nabla u|^{2}+|u|^{2}\right) d x\right\} \\
& \left(R \geq R_{1}+1, u=R(z) f, f \in L_{2}\left(\mathbf{R}^{N}\right), z \in K\right)
\end{aligned}
$$

holds for $\tau$ and $\varepsilon$ satisfying $0<\varepsilon \leq 2 \tau-1$ and $\varepsilon<1$. Here we set

$$
B_{R_{1}, R_{2}}=\left\{x \in \mathbf{R}^{N} / R_{1}<|x|<R_{2}\right\}
$$

and $\sigma_{\varepsilon}$ is as in Notation 3.2.

Proof. It follows from (3.46) that

$$
\begin{aligned}
& \int_{E_{R}}(1+|x|)^{-2 \tau} \sigma_{\varepsilon}\left(|\nabla u|^{2}+|u|^{2}\right) d x \\
& \leq C(1+R)^{-(2 \tau-1-\varepsilon)}\left\{(|f|,|\nabla u|+|u|)_{0}\right. \\
& \left.\quad+\int_{E_{R_{1}}}(1+|x|)^{-2} \sigma_{\varepsilon}\left(|\nabla u|^{2}+|u|^{2}\right) d x\right\}
\end{aligned}
$$


for $R \geq R_{1}+1$ and $u=R(z) f$ with $z \in K$ and $f \in L_{2}\left(\mathbf{R}^{N}\right)$, where $C=C(K)$. Take $R_{2}=R_{2}(K)\left(\geq R_{1}+1\right)$ so that

$$
C\left(1+R_{2}\right)^{-(1-\varepsilon)} \leq \frac{1}{2}
$$

where we should note that $1-\varepsilon>0$. Setting $\tau=1$ and $R=R_{2}$ in (3.60), we get

$$
\begin{aligned}
& \int_{E_{R_{2}}}(1+|x|)^{-2} \sigma_{\varepsilon}\left(|\nabla u|^{2}+|u|^{2}\right) d x \\
& \quad \leq(|f|,|\nabla u|+|u|)_{0}+\int_{B_{R_{1}, R_{2}}}(1+|x|)^{-2} \sigma_{\varepsilon}\left(|\nabla u|^{2}+|u|^{2}\right) d x
\end{aligned}
$$

The estimate (3.58) directly follows from (3.62) and (3.46) with $\tilde{C}=$ $2 C$.

By the use of Proposition 2.3 the second term in the right-hand side of (3.58) can be eliminated.

Proposition 3.10. Let Assumption 1.1 be satisfied. Let $\sigma_{\varepsilon}(r), \tau$, $R_{1}, R_{2}$ and $\varepsilon$ be as above. Let $K$ be a bounded set in $\mathbf{C} \backslash \mathbf{R}$ such that $\operatorname{Re} z \geq \lambda_{0}$ for $z \in K$ with a positive constant $\lambda_{0}$. Then there exists $a$ positive constant $\bar{C}=\bar{C}(K)$ such that

$$
\begin{aligned}
& \int_{E_{R}}(1+|x|)^{-2 \tau} \sigma_{\varepsilon}\left(|\nabla u|^{2}+|u|^{2}\right) d x \\
& \leq \bar{C}(1+R)^{-(2 \tau-1-\varepsilon)}\left(|f|,|\nabla u|^{2}+|u|^{2}\right)_{0} \\
& \quad\left(R \geq R_{1}+1, u=R(z) f, f \in L_{2}\left(\mathbf{R}^{N}\right), \quad z \in K\right) .
\end{aligned}
$$

Proof. By the use of Proposition 2.3 the integral over $B_{R_{1} R_{2}}$ in (3.58) is estimated as

$$
\begin{aligned}
\int_{B_{R_{1} R_{2}}} & (1+|x|)^{-2} \sigma_{\varepsilon}\left(|\nabla u|^{2}+|u|^{2}\right) d x \\
& \leq\left(1+R_{2}\right)^{2 \gamma-2} \int_{R_{1} R_{2}}(1+|x|)^{-2 \gamma}\left(|\nabla u|^{2}+|u|^{2}\right) d x \\
& \leq\left(1+R_{2}\right)^{2 \gamma-2} C(|f|,|\nabla u|+|u|)_{0},
\end{aligned}
$$

where the constants $\gamma$ and $C$ are as given in Proposition 2.3. The estimate (3.63) is obtained from (3.58) and (3.64) by noting that $R_{2}=$ $R_{2}(K)$ depend only on $K$.

4. The limiting absorption principle. The results obtained in the preceding section will be used to show the limiting absorption principle for the operator $H$. 
Theorem 4.1. Suppose that Assumptions 1.1 are satisfied. Let $K$ be a bounded set of $\mathbf{C} \backslash \mathbf{R}$ such that we have for $z=\lambda+i \eta \in K$

$$
\lambda \geq \lambda_{0}>0
$$

with a positive number $\lambda_{0}$. Let $\delta$ be a real number such that

$$
\delta>1 / 2 \text {. }
$$

Then there exists a positive constant $C=C(K, \delta)$ such that

$$
\|R(z) f\|_{2,-\delta} \leq C\|f\|_{\delta}
$$

holds for any $f \in L_{2, \delta}\left(\mathbf{R}^{N}\right)$ and any $z \in K$, where $R(z)$ is the resolvent of $H$.

Proof. (I) Let us first show that

$$
\|R(z) f\|_{1,-\delta} \leq C\|f\|_{\delta} \quad\left(z \in K, f \in L_{2, \delta}\left(\mathbf{R}^{N}\right)\right) .
$$

We can assume with no loss of generality that

$$
1 / 2<\delta \leq 1
$$

Let us assume (4.4) is false. Then there exist sequences $\left\{z_{m}\right\} \subset K$ and $\left\{f_{m}\right\} \subset L_{2, \delta}\left(\mathbf{R}^{N}\right)$ such that

$$
\left\|\mathbf{R}\left(z_{m}\right) f_{m}\right\|_{1,-\delta}=1 \text { and }
$$$$
\left\|f_{m}\right\|_{\delta}<1 / m
$$$$
(m=1,2, \ldots)
$$

We shall set

$$
u_{m}=R\left(z_{m}\right) f_{m}
$$

Since the sequence $\left\{z_{m}\right\}$ is contained in a bounded subset $K$ of $\mathbf{C}$, there exists a subsequence of $\left\{z_{m}\right\}$ which converges to an element $z_{0}$ of $\mathbf{C}$. For the sake of avoiding complication of notations we shall express the subsequence by $\left\{z_{m}\right\}$ again. Since $u_{m}$ satisfies the equation

$$
-\Delta u_{m}-z_{m} \mu(x) u_{m}=\mu(x) f_{m},
$$

it follows from the Rellich Lemma and the interior estimate that there exists a subsequence of $\left\{u_{m}\right\}$ which converges to $u_{0}$ in $H^{1}\left(\mathbf{R}^{N}\right)_{\text {loc }}$. The subsequence will be denoted by $\left\{u_{m}\right\}$ again.

Set $\tau=\delta, \varepsilon=(2 \delta-1) / 2, u=u_{m}$, and $f=f_{m}$ in (3.63). Here we should note that the conditions $2 \tau-1 \geq \varepsilon>0$ and $\varepsilon<1$ are satisfied. Then we have

$$
\begin{aligned}
& \int_{E_{R}}(1+|x|)^{-2 \delta} \sigma_{\varepsilon}\left(\left|\nabla u_{m}\right|^{2}+\left|u_{m}\right|^{2}\right) d x \\
& \quad \leq \bar{C}(1+R)^{-(2 \delta-1) / 2}\left(\left|f_{m}\right|,\left|\nabla u_{m}\right|+\left|u_{m}\right|\right)_{0} \quad(m=1,2, \ldots)
\end{aligned}
$$


for any $R \geq R_{1}+1$, where $R_{1}$ is given in Lemma 3.6. Note that
(4.10)
$0<\exp \left\{-\varepsilon^{-1}\left(1+R_{1}\right)^{-\varepsilon}\right\} \leq \sigma_{\varepsilon}(r) \leq 1$
$\left(r \geq R_{1}, \varepsilon>0\right)$,

and the term $\left(\left|f_{m}\right|,\left|\nabla u_{m}\right|+\left|u_{m}\right|\right)$ is uniformly bounded for $n=1,2, \ldots$ by (4.6) and the Schwarz inequality. Thus it follows from (4.9) and (4.10) that

$$
\left\|u_{m}\right\|_{1,-\delta, E_{R}} \leq C_{1}(1+R)^{-(2 \tau-1) / 2} \quad(m=1,2, \ldots)
$$

with a constant $C_{1}$, where \|\|$_{1,-\delta, E_{R}}$ is the norm of $H_{-\delta}^{1}\left(E_{R}\right)$ with $E_{R}=\left\{x \in \mathbf{R}^{N} /|x|>R\right\}$. Thus the (sub)sequence $\left\{u_{m}\right\}$ not only converges to $u_{0}$ in $H^{1}\left(\mathbf{R}^{N}\right)_{\text {loc }}$ but also has a uniformly small $H_{-\delta}^{1}\left(E_{R}\right)$ norm when $R \rightarrow \infty$. Therefore we have shown that the sequence $\left\{u_{m}\right\}$ converges to $u_{0}$ in $H_{-\delta}^{1}\left(\mathbf{R}^{N}\right)$. Especially we have

$$
\left\|u_{0}\right\|_{1,-\delta}=1 \text {. }
$$

Let $m \rightarrow \infty$ and $R=R_{1}+1$ in (4.9). Then, since $\left\{u_{m}\right\}$ is proved to converge to $u_{0}$ in $H_{-\delta}^{1}\left(\mathbf{R}^{N}\right)$, in the left-hand side of (4.9) we have

$$
\begin{aligned}
\lim _{m \rightarrow \infty} & \int_{E_{R_{1}+1}}(1+|x|)^{-2 \delta} \sigma_{\varepsilon}\left(\left|\nabla u_{m}\right|^{2}+\left|u_{m}\right|^{2}\right) d x \\
& =\int_{E_{R_{1}+1}}(1+|x|)^{-2 \delta} \sigma_{\varepsilon}\left(\left|\nabla u_{0}\right|^{2}+\left|u_{0}\right|^{2}\right) d x .
\end{aligned}
$$

On the other hand, by (4.6) and the Schwarz inequality, the right-hand side of (4.9) converges to zero as $m \rightarrow \infty$. Therefore we arrive at

$$
u_{0}=0 \text { on } E_{R_{1}+1} \text {, }
$$

which implies, by noticing that $u_{0}$ is a solution of the elliptic equation $-\Delta u-z_{0} \mu(x) u=0$ and by the use of the unique continuation theorem, that $u_{0}$ is identically zero on $\mathbf{R}^{N}$. This contradicts (4.12). Thus we have proved (4.4).

(II) It is easily seen that (4.3) follows from (4.4) and the fact that $u$ is a solution of the equation $-\Delta u-k^{2} u=\mu(x) f$.

REMARK 4.2 As we have seen, Proposition 3.10 plays a crucial role in the above proof of Theorem 4.1, and Proposition 3.10 is an improvement of Corollary 3.9 by Proposition 2.3. It might be interesting to discuss what we can get if we use only Corollary 3.9 without using Proposition 2.3. Let us suppose that (4.6) holds with $\left\{z_{m}\right\} \in K$, $\left\{f_{m}\right\} \subset L_{2, \delta}\left(\mathbf{R}^{N}\right)$ and $\left\{u_{m}\right\}=\left\{R\left(z_{m}\right) f_{m}\right\}$. We can assume, with no loss of generality, that the sequence $\left\{z_{m}\right\}$ converges to a positive number $\lambda_{1}$ (if the limit is a nonreal number, a contradiction follows much 
more easily). It follows from the estimate (3.58) in Corollary 3.9 that there exists a subsequence of $\left\{u_{m}\right\}$, which will be denoted by $\left\{u_{m}\right\}$ again, such that

$$
\text { s- } \lim _{m \rightarrow \infty} u_{m}=u_{0} \quad \text { in } H_{-\tau}^{1}\left(\mathbf{R}^{N}\right)
$$

for any $\tau>1 / 2$. As has been shown, $u_{0}$ is a solution of the homogeneous equation $-\Delta u-\lambda_{1} \mu(x) u=0$.

Let $\varepsilon$ satisfy $0<\varepsilon \leq 1$ and set $\tau=(1+\varepsilon) / 2, u=u_{m}, f=f_{m}$ and $R=R_{*}=\operatorname{Max}\left\{R_{1}+1, R_{2}\right\}$ and (3.58). Then we have

$$
\begin{aligned}
& \text { (4.16) } \int_{E_{R_{*}}}(1+|x|)^{-(1+\varepsilon)} \sigma_{\varepsilon}\left(\left|\nabla u_{m}\right|^{2}+\left|u_{m}\right|^{2}\right) d x \\
& \leq C^{\prime}\left\{\left(\left|f_{m}\right|,\left|\nabla u_{\mu}\right|^{2}+\left|u_{m}\right|^{2}\right)_{0}\right. \\
& \left.+\int_{B_{R_{1} R_{2}}}(1+|x|)^{-2} \sigma_{\varepsilon}\left(\left|\nabla u_{m}\right|^{2}+\left|u_{m}\right|^{2}\right) d x\right\}
\end{aligned}
$$

with a constant $C^{\prime}$ which is independent of $\varepsilon$ in $(0,1)$. Let $m \rightarrow \infty$ in (4.16). Then we get

$$
\begin{aligned}
\int_{E_{R_{*}}} & (1+|x|)^{-(1+\varepsilon)} \sigma_{\varepsilon}\left(\left|\nabla u_{0}\right|^{2}+\left|u_{0}\right|^{2}\right) d x \\
& \leq C^{\prime} \int_{B_{R_{1} R_{2}}}(1+|x|)^{-2} \sigma_{\varepsilon}\left(\left|\nabla u_{0}\right|^{2}+\left|u_{0}\right|^{2}\right) d x .
\end{aligned}
$$

By noting that

$$
\sigma_{\varepsilon}(r) \geq \sigma_{\varepsilon}\left(R_{*}\right) \geq \sigma_{\varepsilon}\left(R_{2}\right)=\exp \left\{-\varepsilon^{-1}\left(1+R_{2}\right)^{-1}\right\} \quad\left(r \geq R_{*}\right)
$$

and

$$
\sigma_{\varepsilon}\left(R_{2}\right)^{-1} \cdot \sigma_{\varepsilon}(r) \leq 1 \quad\left(R_{1} \leq r \leq R_{2}\right),
$$

it follows form (4.17) that

$$
\begin{aligned}
\int_{E_{R_{*}}} & (1+|x|)^{-(1+\varepsilon)}\left(\left|\nabla u_{0}\right|^{2}+\left|u_{0}\right|^{2}\right) d x \\
& \leq C^{\prime} \int_{B_{R_{1} R_{2}}}(1+|x|)^{-2}\left(\left|\nabla u_{0}\right|^{2}+\left|u_{0}\right|^{2}\right) d x
\end{aligned}
$$

for any $\varepsilon$ in $(0,1)$, whence we get, by letting $\varepsilon$ to zero,

$$
\int_{E_{R_{*}}}(1+|x|)^{-1}\left(\left|\nabla u_{0}\right|^{2}+\left|u_{0}\right|^{2}\right) d x<\infty,
$$

i.e., we have $u_{0} \in H_{-1 / 2}^{1}\left(\mathbf{R}^{N}\right)$. 
If $u_{0}$ is a solution of the homogeneous Schrödinger equation

$$
-\Delta u+V u-\lambda_{1} u=0 \quad\left(\lambda_{1}>0\right)
$$

with, e.g., a short-range or long-range potential $V(x)$, then, by the theorems on the asymptotic behavior of a solution of the equation (4.22) (e.g., Kato [7], Eastham \& Kalf [3]), the estimate (4.21) is sufficient to guarantee that $u_{0}$ is identically zero. However, it seems to be an open problem whether the above theorems on the asymptotic behavior of the solution can be extended to our case.

Now that the estimate (4.3) has been shown, we can make use of Theorem 1.2 to show that the limiting absorption principle for the operator $H$ holds between $L_{2, \delta}\left(\mathbf{R}^{N}\right)$ and $L_{2,-\delta}\left(\mathbf{R}^{N}\right)$.

Theorem 4.3. Suppose that Assumptions 1.1 are satisfied. Let $R(z)$ be the resolvent of the selfadjoint operator $H$ and let $\delta>1 / 2$. Then for each $\lambda>0$ there exist the operators $R^{ \pm}(\lambda)$ in $B\left(L_{2, \delta}\left(\mathbf{R}^{N}\right), L_{2,-\delta}\left(\mathbf{R}^{N}\right)\right)$ such that we have

$$
\text { s- } \lim _{\eta \rightarrow \pm 0} R(\lambda+i \eta) f=R^{ \pm}(\lambda) f \quad \text { in } H_{-\delta}^{2}\left(\mathbf{R}^{N}\right)
$$

for all $f \in L_{2, \delta}\left(\mathbf{R}^{N}\right)$. Furthermore, $R^{ \pm}(\lambda)$ is an $H_{-\delta}^{2}\left(\mathbf{R}^{N}\right)$-valued, strongly continuous function on $(0, \infty)$ for each $f \in L_{2, \delta}\left(\mathbf{R}^{N}\right)$.

Proof. It is sufficient to assume that $1 / 2<\delta \leq 1$. Let us show the existence of the limit (4.23) only when $\eta \rightarrow+0$. The case where $\eta \rightarrow-0$ can be treated in the same way. Let us denote by $R^{+}(\lambda)$ the operator in $B\left(L_{2,1}\left(\mathbf{R}^{N}\right), L_{2,-1}\left(\mathbf{R}^{N}\right)\right)$ whose existence has been proved by Theorem 1.2. Thus we have

$$
\text { s- } \lim _{\eta \rightarrow+0} R(\lambda+i \eta) f=R^{+}(\lambda) f \quad \text { in } L_{2,-1}\left(\mathbf{R}^{N}\right)
$$

for all $f \in L_{2,1}\left(\mathbf{R}^{N}\right)$.

Let us first prove (4.23) for $f \in L_{2,1}\left(\mathbf{R}^{N}\right)$. Let $\left\{\eta_{m}\right\}$ be an arbitrary positive sequence such that $\eta_{m} \rightarrow+0$ and let $u_{m}=R\left(z_{m}\right) f$ with $z_{m}=\lambda+i \eta_{m}$. Then, proceeding as in the proof of Theorem 4.1, we can find a subsequence $\left\{u_{m_{j}}\right\}$ of $\left\{u_{m}\right\}$ which converges strongly to an element $u_{0}$ in $H_{-\delta}^{2}\left(\mathbf{R}^{N}\right)$ with the estimate

$$
\left\|u_{0}\right\|_{2,-\delta} \leq C\|f\|_{\delta}
$$

with a positive constant $C$ which remains bounded when $\lambda$ moves in a compact set in $(0, \infty)$. On the other hand it follows from (4.24) that 
the sequence $\left\{u_{m}\right\}$ itself converges to $R^{+}(\lambda) f$ in $L_{2,-1}\left(\mathbf{R}^{N}\right)$. Therefore we have $u_{0}=R^{+}(\lambda) f$,

$$
\text { s- } \lim _{j \rightarrow \infty} u_{m_{j}}=R^{+}(\lambda) f \quad \text { in } H_{-\delta}^{2}\left(\mathbf{R}^{N}\right),
$$

and

$$
\left\|R^{+}(\lambda) f\right\|_{2,-\delta} \leq C\|f\|_{\delta} .
$$

Since the sequence $\left\{\eta_{m}\right\}$ was taken arbitrarily, we can conclude that (4.23) is true for $f=L_{2,1}\left(\mathbf{R}^{N}\right)$. It follows from (4.27) and the denseness of $L_{2,1}\left(\mathbf{R}^{N}\right)$ in $L_{2, \delta}\left(\mathbf{R}^{N}\right)$ that $R^{+}(\lambda)$ can be uniquely extended to a bounded linear operator from $L_{2, \delta}\left(\mathbf{R}^{N}\right)$ into $H_{-\delta}^{2}\left(\mathbf{R}^{N}\right)$. The extension will be denoted by $R^{+}(\lambda)$ again. Then, by use of the denseness of $L_{2,1}\left(\mathbf{R}^{N}\right)$ in $L_{2, \delta}\left(\mathbf{R}^{N}\right)$ and the estimates (4.3) and (4.27), it is easy to see that (4.23) is true for $f \in L_{2, \delta}\left(\mathbf{R}^{N}\right)$. Noting that $u=R^{+}(\lambda) f$ satisfies the estimate (3.63) as well as (4.27), we can almost repeat the above arguments to show the continuity of $R^{+}(\lambda) f$ in $H_{-\delta}^{2}\left(\mathbf{R}^{N}\right)$ with respect to $\lambda$ for any $f \in L_{2}\left(\mathbf{R}^{N}\right)$.

\section{REFERENCES}

[1] S. Agmon, Spectral properties of Schrödinger operators and scattering theory, Ann. Scoula Nor. Sup. Pisa, (4) 2 (1975), 151-218.

[2] A. Devinatz and P. Rejto, A limiting absorption principle for Schrödinger operators with oscillating potentials. I, II, J. Diff. Equations, 49 (1983), 29-84, 85-104.

[3] M. S. P. Eastham and H. Kalf, Schrödinger-Type Operators With Continuous Spectra, Pitman, London, 1982.

[4] D. Eidus, The limiting absorption and amplitude principles for the diffraction problem with two unbounded media, Comm. Math. Phys., 107 (1986), 29-38.

[5] T. Ikebe and Y. Saitō, Limiting absorption method and absolute continuity for the Schrödinger operator, J. Kyoto Univ., 7 (1972), 513-542.

[6] W. Jäger, Ein gewöhnlicher Differentialoperator zweiter Ordnung für Funktionen mit Werten in einem Hilbertraum, Math. Z., 113 (1970), 68-98.

[7] T. Kato, Growth properties of solutions of the reduced wave equation with a variable coefficient, Comm. Pure Appl. Math., 12 (1959), 403-425.

[8] R. Lavine, Absolute continuity of positive spectrum for Schrödinger operators with long-range potentials, J. Funct. Anal., 12 (1973), 30-54.

[9] K. Mochizuki and J. Uchiyama, Radiation conditions and spectral theory for 2-body Schrödinger operators with "oscillatory" long-range potentials, I. J. Math. Kyoto Univ., 18 (1978), 377-408.

[10] Y. Saitō, The principle of limiting absorption for second-order differential equations with operator-valued coefficients, Publ. Res. Inst. Math. Sci. Kyoto Univ., 7 (1972/72), 581-619.

[11] Spectral representations for Schrödinger operators with long-range potentials, Lecture Notes in Mathematics 727, Springer, Berlin-Heidelberg-New York, 1979. 
[12] Schrödinger operators with nonspherical radiation condition, Pacific J. Math., 126 (1987), 331-359.

Received August 24, 1987 and in revised form March 18, 1988. Partially supported by NSF EPSCoR grant number RII-8610669.

UNiversity of Alabama AT BiRmingham

BIRMINGHAM, AL 35294 


\section{PACIFIC JOURNAL OF MATHEMATICS EDITORS}

\author{
V. S. VARADARAJAN \\ (Managing Editor) \\ University of California \\ Los Angeles, CA 90024 \\ HeRbert Clemens \\ University of Utah \\ Salt Lake City, UT 84112 \\ THOMAS ENRIGHT \\ University of California, San Diego \\ La Jolla, CA 92093
}

R. FINN

Stanford University

Stanford, CA 94305

HERMANN FLASCHKA

University of Arizona

Tucson, AZ 85721

VAUGHAN F. R. JONES

University of California

Berkeley, CA 94720

STEVEN KERCKHOFF

Stanford University

Stanford, CA 94305

\section{ROBION KIRBY}

University of California

Berkeley, CA 94720

C. C. MOORE

University of California

Berkeley, CA 94720

HAROLD STARK

University of California, San Diego

La Jolla, CA 92093

\section{ASSOCIATE EDITORS}
R. ARenS
E. F. BECKENBACH
B. H. NeumanN
F. WOLF
K. YOSHIDA (1906-1982)

\section{SUPPORTING INSTITUTIONS}
UNIVERSITY OF ARIZONA
UNIVERSITY OF OREGON
UNIVERSITY OF BRITISH COLUMBIA
UNIVERSITY OF SOUTHERN CALIFORNIA
CALIFORNIA INSTITUTE OF TECHNOLOGY
STANFORD UNIVERSITY
UNIVERSITY OF CALIFORNIA
MONTANA STATE UNIVERSITY
UNIVERSITY OF HAWAII
UNIVERSITY OF NEVADA, RENO
UNIVERSITY OF TOKYO
NEW MEXICO STATE UNIVERSITY
UNIVERSITY OF UTAH
OREGON STATE UNIVERSITY
WASHINGTON STATE UNIVERSITY
UNIVERSITY OF WASHINGTON 


\section{Pacific Journal of Mathematics}

\section{Vol. 136, No. $1 \quad$ November, 1989}

Robert Archbold and Frederic W. Shultz, Characterization of $C^{*}$-algebras with continuous trace by properties of their pure states $\ldots \ldots \ldots \ldots \ldots \ldots 1$

Shu Ping Chen and Roberto Triggiani, Proof of extensions of two conjectures on structural damping for elastic systems $\ldots \ldots \ldots \ldots \ldots \ldots$

Philip Throop Church and James Timourian, A nonlinear elliptic

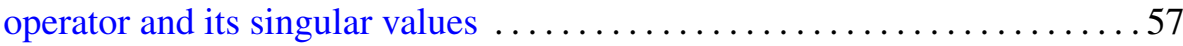

A. Gervasio Colares and Katsuei Kenmotsu, Isometric deformation of surfaces in $R^{3}$ preserving the mean curvature function $\ldots \ldots \ldots \ldots \ldots 71$

Fei Xu, A remark on spinor norms of local integral rotations. I . . . . . . . 81

Pedro Martinez Gadea and Ángel María Montesinos-Amilibia, Spaces of constant para-holomorphic sectional curvature $\ldots \ldots \ldots \ldots \ldots \ldots \ldots 5$

Guangxin Zeng, Homogeneous Stellensätze in semialgebraic geometry . . . . 103

Thomas Eric Hall, The isomorphism problem for orthodox semigroups . . . . 123

Mike Hoffman, Noncoincidence index, free group actions, and the fixed

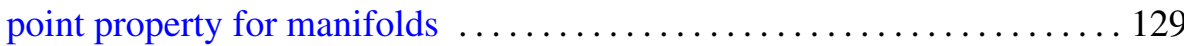

Terry Atherton Loring, The noncommutative topology of one-dimensional

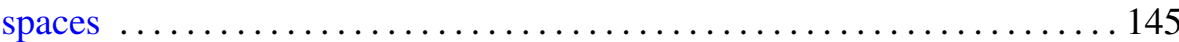

Haskell Paul Rosenthal and Alan Evan Wessel, The Krě̆ n-Mil'man property and a martingale coordinatization of certain nondentable convex sets

Yoshimi Saito, A remark on the limiting absorption principle for the reduced wave equation with two unbounded media 